\title{
Práxis das equipes saúde da família no cuidado com paciente oncológico
}

Praxis of family health teams in the care of cancer patients Praxis de los equipos de Unidades de Salud de la Familia en el cuidado de pacientes oncológicos

Letícia Flores Trindade ${ }^{1,2}$ (c) https://orcid.org/000-0001-9088-2825 Adriane Cristina Bernat Kolankiewicz' io https://orcid.orgy/0000-0003-1793-7783

Laura Renner Bandeira ${ }^{1}$ iD https://orcid.org/0000-0002-4788-3942

Caroline Donini Rodrigues ${ }^{1}$ iD https://orcid.org/0000-0003-0753-2862 Regina Célia Gollner Zeitoune ${ }^{3}$ io https://orcid.org/0000-0002-0276-8166 Marli Maria Loro ${ }^{1}$ id https://orcid.org/0000-0003-2731-5224

Como citar: Trindade LF, Kolankiewicz AC, Bandeira LR, Rodrigues $\mathrm{CD}$, Zeitoune RC, Loro MM. Práxis das equipes saúde da família no cuidado com paciente oncológico. Acta Paul Enferm. 2021;34:eAPE03054.

DOI

http://dx.doi.org/10.37689/actaape/2021A003054

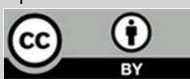

Descritores

Atenção primária à saúde; Estratégia saúde da família; Oncologia; Pesquisa sobre serviços de saúde; Saúde da família

Keywords

Family health strategy; health services research; medical oncology; primary health care; Family

Descriptores

Atención primaria de salud; Estrategia de salud familiar; Oncología médica; Investigación sobre servicios de salud; Salud de la família

Submetido 15 de Novembro de 2019

Aceito

1 de Março de 2021

Autor correspondente Adriane Cristina Bernat Kolankiewicz E-mail: adri.saudecoletiva@gmail.com

\section{Resumo}

Objetivo: Refletir acerca dos atributos da Atenção Primária à Saúde e identificar potencialidades e fragilidades do cuidado efetivado ao paciente oncológico.

Métodos: Estudo do tipo pesquisa-ação, realizado por meio de oficinas educativas, com profissionais que atuavam em cinco unidades de saúde da família. Os dados foram coletados no período de janeiro a setembro de 2017, e seu tratamento se deu por análise de conteúdo.

Resultados: Considerando os atributos da Atenção Primária à Saúde, os trabalhadores da Estratégias Saúde da Família refletiram sobre a práxis assistencial, emergindo a categoria temática "Saberes e práticas de profissionais de saúde na assistência oncológica: um olhar sobre a realidade".

Conclusão: Implementar uma prática educativa possibilitou pautar aspectos importantes sobre 0 cuidado efetivo ao paciente oncológico. Ainda, utilizar a metodologia embasada na pesquisa-ação teve potencial de produzir nos trabalhadores reflexões sobre o fazer assistencial e, na mesma medida, identificar potencialidades e fragilidades, o que implicou em analisá-las e superá-las.

\section{Abstract}

Objective: To reflect on the attributes of Primary Health Care and identify the strengths and weaknesses of the care provided to cancer patients.

Methods: Action-research study, performed through educational workshops with professionals who worked in five family health units. Data were collected from January to September 2017, and data treatment was based on content analysis.

Results: Considering the attributes of Primary Health Care, the Family Health Strategies workers reflected on the practice of assistance. The analysis gave rise to the thematic category "Knowledge and practices of health professionals in cancer care: a look at reality".

Conclusion: The implementation of an educational practice made it possible to determine important aspects about effective care for cancer patients. The use of the method had the potential to encourage reflections on the assistance provided and help professionals to identify weaknesses and analyze and try to overcome them.

\section{Resumen}

Objetivo: Reflexionar sobre los atributos de la Atención Primaria de Salud e identificar posibilidades y debilidades de los cuidados realizados a pacientes oncológicos.

Universidade Regional do Noroeste do Estado do Rio Grande do Sul, ljuí, RS, Brasi.

2Universidade de Cruz Alta, ljuí, RS, Brasil.

${ }^{3}$ Escola de Enfermagem Anna Nery, Universidade Federal do Rio de Janeiro, Rio de Janeiro, RJ, Brasil.

Conflitos de interesse: nada a declarar. 
Métodos: Estudio tipo investigación-acción, realizado mediante talleres educativos, con profesionales que actuaban en cinco Unidades de Salud de la Familia. Los datos fueron recopilados durante el período de enero a septiembre de 2017 y fueron tratados mediante el análisis de contenido.

Resultados: Considerando los atributos de la Atención Primaria de Salud, los trabajadores de la Estrategia Salud de la Familia reflexionaron sobre la praxis asistencial, donde surgió la categoría temática "Saberes y prácticas de profesionales de la salud en la atención oncológica: una mirada sobre la realidad".

Conclusión: Implementar una práctica educativa permitió enumerar aspectos importantes sobre los cuidados realizados a pacientes oncológicos. Además, utilizar la metodología basada en la investigación-acción permitió que los trabajadores reflexionaran sobre la práctica asistencial e identificaran, en la misma medida, posibilidades y debilidades, y como consecuencia, analizarlas y superarlas.

\section{Introdução}

O reconhecimento das condiçôes de saúde de uma população é fator indispensável para um cuidado resolutivo e de qualidade. A condição de saúde é compreendida além da instalação de doenças prévias, pois incorpora condiçóes fisiológicas e o acompanhamento longitudinal dos ciclos de vida dos indivíduos. ${ }^{(1)}$ Ainda, os modelos de atenção à saúde são categorizados em condiçóes agudas e crônicas. ${ }^{(2)}$

Tais condiçôes de saúde são de responsabilidade da Atenção Primária à Saúde, sendo importante que, dentre elas, seja dada maior visibilidade às crônicas, em maior magnitude destas no território brasileiro. Entre os principais fatores que ocasionaram o aumento relativo das condiçóes crônicas estão as transformações demográficas advindas do envelhecimento populacional, a industrializaçáo, a urbanização acelerada e as mudanças nos padróes de consumo e nos estilos de vida. ${ }^{(3)}$

$\mathrm{O}$ rápido processo de envelhecimento da população coloca em evidência algumas doenças crônicas, como o câncer. No Brasil, a mortalidade proporcional por câncer cresceu consideravelmente nas últimas décadas, conforme o cenário mundial, ${ }^{(4)}$ sendo caracterizada como um problema de saúde pública. ${ }^{(3)}$ Nesse ínterim, a doença diferencia-se das demais enfermidades, pois a incerteza do prognóstico, os efeitos dos tratamentos e o enfrentamento da possibilidade de recidiva e morte encontram-se ligados a fatores presentes em todos os estágios da doença. ${ }^{(5)}$

O auxílio na vivência do processo de doença, na conduta clínica e na reabilitação de todos os envolvidos no processo do câncer perfaz as açóes das equipes de saúde. ${ }^{(6)}$ Nessa perspectiva, no cenário brasileiro, a organização dos cuidados em saúde, nos diversos níveis de complexidade, é coordenado pela Atenção Primária à Saúde, é entendida como principal porta de entrada das Rede de Atençáo à Saúde e responsável pela identificação dos problemas de saúde da população, sendo transversal a todos os níveis do Sistema Único de Saúde (SUS). ${ }^{(7)}$

Ao reconhecer a importância da Atenção Primária à Saúde para a efetividade dos sistemas de saúde, com vistas a desempenhar o papel de organizadora do sistema e coordenadora do cuidado, propóem-se atributos essenciais e norteadores do serviço. Sáo esses atributos: Acesso de Primeiro Contato, Longitudinalidade, Integralidade e Coordenaçáo e os derivados Orientação Familiar e Comunitária. (8) Eles podem ser mensurados pelo instrumento Primary Care Assessment Tool (PCATool), o qual permite identificar aspectos dos serviços que exigem reafirmação ou reformulação, visando à qualidade da assistência. ${ }^{(9)}$

Essa utilização destes atributos, permite que as açóes e os serviços desempenhados pela Atenção Primária à Saúde sejam qualificados e centrados na família. Cabe ainda às equipes de Estratégia Saúde da Família refletirem sobre suas atividades e condutas diárias, a fim de avaliá-las e fomentar mudanças, por meio do olhar crítico dos profissionais, permitindo as discussóes de boas práticas em saúde. ${ }^{(10)}$

Desenvolver açóes educativas, com equipes de saúde, em um processo crítico-reflexivo acerca de sua práxis, tem o potencial de qualificar a assistência aos indivíduos e à comunidade. ${ }^{(1)}$ Assim, justifica-se a realização deste estudo, uma vez que, dada a incidência e a prevalência do câncer na contemporaneidade, observa-se a necessidade de averiguar como os pacientes oncológicos e os profissionais de saúde percebem a presença e a extensão dos atributos essenciais e derivados da Atenção Primária à Saúde e como os profissionais de saúde notam sua práxis cotidiana na unidade, ao efetivar o cuidado.

O objetivo deste estudo foi refletir acerca dos atributos da Atenção Primária à Saúde e identificar 
potencialidades e fragilidades do cuidado efetivado ao paciente oncológico.

\section{Métodos}

Trata-se de estudo qualitativo, pautado na modalidade pesquisa-ação. Foi desenvolvido em duas etapas: a primeira foi um recorte de projeto institucional denominado Demandas de Cuidado de Pacientes Oncológicos em Tratamento: Proposta de Intervenção pela Convergência da Pesquisa e Prática Educativa.

Dessa etapa, participaram 268 pacientes oncológicos em tratamento, assistidos na Atenção Primária à Saúde de um município situado no noroeste do estado do Rio Grande do Sul, selecionados a partir de uma lista de nomes e endereços obtida nas unidades de saúde. Seus dados foram coletados em seus domicílios. A seleção dos pacientes ocorreu por conveniência.

Participaram ainda 15 profissionais de saúde, que foram entrevistados em suas unidades, em horário previamente agendado. Dentre os entrevistados, estavam gestores municipais de saúde, enfermeiros e médicos atuantes nas Estratégia Saúde da Família do mesmo município em questão.

Para obtençáo dos dados, utilizou-se o instrumento autoaplicável PCATool, para os pacientes e profissionais. ${ }^{(12)} \mathrm{O}$ PCATool mede a presença e a extensão dos quatro atributos essenciais (Acesso de Primeiro Contato, Longitudinalidade, Coordenaçáo - integração de cuidados e sistema de informação e Integralidade - serviços disponíveis e prestados) e dos dois atributos derivados da Atenção Primária à Saúde (orientação familiar e comunitária). O instrumento é validado para a realidade brasileira, nas versōes para usuários ${ }^{(13)}$ e profissionais. ${ }^{(14)}$

Os dados foram organizados no programa Epi $\operatorname{Info}^{\text {тм }}$ 6.04, com dupla digitaçáo independente. Após correçóes de erros e inconsistências, a análise estatística foi realizada no programa Statistical Package for the Social Sciences (SPSS) ${ }^{\circ}$, versão 25.0. Para análise do PCATool, foram calculados os escores e os valores das perguntas de cada atributo, os quais variam em uma escala de 1 a 4 , e o escore final de cada um deles é dado pela média das respostas de suas perguntas. Para avaliação de alto e baixo escore dos atributos, foram utilizados valores de escores $\geq 6,6$, definindo resultados iguais ou superior a esse como extensão adequada (satisfatória) e equivalentes ao valor 3 ou mais na escala Likert. ${ }^{(9)}$

Tendo em vista o valor de escore considerado satisfatório, a avaliação da Atenção Primária à Saúde perante a ótica dos profissionais foi: 7,14 para Acesso de Primeiro Contato; 3,42 para Longitudinalidade; 7,67 para Coordenação - integração dos cuidados, 7,49 para Coordenação - sistema de informaçáo; 7,63 para Integralidade - serviços disponíveis, 7,17 para Integralidade - serviços prestados; 7,08 para Orientação familiar e 7,33 para Orientação Comunitária.

$\mathrm{Na}$ percepção de usuários oncológicos, os resultados foram 6,96 para Acesso de Primeiro Contato - utilidade, 3,36 para Acesso de Primeiro Contato - acesso, 7,21 para Longitudinalidade, 4,12 para Coordenação - integraçáo dos cuidados, 7,02 para Coordenação - sistema de informação, 5,80 para Integralidade - serviços disponíveis, 3,77 para Integralidade - serviços prestados, 4,28 para Orientação familiar e 5,46 para Orientação Comunitária.

Assim, acessou-se a segunda etapa, que sistematizou a efetivação das oficinas educativas. Participaram da segunda etapa cinco equipes de saúde.

As oficinas foram realizadas entre os meses de janeiro a setembro de 2017. O enfermeiro gestor da unidade foi contatado para agendamento de data e horário da atividade, de modo que toda a equipe pudesse se fazer presente.

As oficinas foram planejadas com o intuito de estimular o diálogo em grupo, para abstrair seus saberes e compreensóes sobre a problemática, bem como estimular a avaliaçáo de seus serviços e propor meios e/ou medidas para o fortalecimento da atenção em saúde ao paciente oncológico. Cada encontro teve a duração média de 1 hora e 30 minutos, e o número variou entre um a dois encontros, por unidade, conforme a participação da equipe e o esgotamento do tema em tela.

Os resultados da primeira etapa foram apresentados de forma a estimular sua discussão e a reflexão 
da práxis efetivada a estes pacientes, bem como a possibilitar pactuar açóes com potencial de qualificar a assistência. Nessas oficinas, foram convidados a participar todos os profissionais que atuavam na Estratégia Saúde da Família, pois compreende-se que o cuidado com paciente oncológico é efetivado por toda a equipe.

Com vistas a observar os preceitos éticos, as unidades de Estratégia Saúde da Família foram identificadas pelos números 1 a 5 . Os participantes foram identificados por letras que representavam a categoria profissional: ENF para enfermeiro, MED para médico, NUT para nutricionista, TEC. ENF para técnico de enfermagem e ACS agente comunitário de saúde. Os depoimentos dos participantes foram gravados e, posteriormente, transcritos na íntegra.

A análise e a interpretação incluiu a transcrição dos relatos dos profissionais das oficinas, para que fosse possível a categorização por meio da análise temática de conteúdo. ${ }^{(15)}$ Aos dados foram aplicadas as técnicas de análise de conteúdo temática, da qual emergiu a seguinte categoria: "Saberes e práticas de profissionais de saúde na assistência oncológica: um olhar sobre a realidade".

Estudo aprovado pelo Comitê de Ética em Pesquisa, sob CAAE: 03261712.8.0000.5505.

\section{Resultados e discussão}

$\mathrm{Na}$ efetivação das oficinas educativas, a equipe refletiu e avaliou os atributos essenciais e derivados da Atenção Primária à Saúde, de forma a elucidar o que levou os profissionais e os pacientes a atribuírem tais valores de escores a cada atributo, definindo estratégias com vistas a superação de fragilidades e qualificação da prática assistencial.

\section{Saberes e práticas de profissionais de saúde na assistência oncológica: um olhar sobre a realidade}

$\mathrm{Na}$ contemporaneidade, a emergência das condições crônicas, especialmente do câncer, torna necessário que as equipes de saúde, atuantes nas Estratégia Saúde da Família, dispensem cuidado à saúde desses indivíduos, centrados nos atributos norteadores da Atenção Primária à Saúde. Logo, eles representam importantes indicadores de qualidade da assistência prestada. ${ }^{(16)}$
À luz desse contexto, a pesquisa-ação é uma metodologia que propicia interação e/ou cooperação mútua entre o pesquisador e o pesquisado, de forma que se estabeleça uma relação participativa e ativa com o coletivo. ${ }^{(17)}$ Ainda, entende-se que sua utilização no contexto da Estratégia Saúde da Família torna-se importante, tendo em vista que, nesse local, já há interação prévia entre pesquisador e pesquisado, por meio de relação já estabelecida pelos profissionais da saúde e comunidade, na qual a equipe permite diálogo aberto, participativo e reflexivo com seus clientes, na busca constante pela transformação positiva. ${ }^{(18)}$

O Acesso de Primeiro Contato é definido como a porta de entrada dos usuários nos serviços de saúde, identificando-o como o primeiro recurso a ser buscado quando há uma necessidade ou problema de saúde. ${ }^{(19)}$ A visualização dos escores fez com que os participantes buscassem hipóteses dos motivos que levaram à avaliação por vezes positiva e/ou negativa. $\mathrm{O}$ grupo em interação aludiu que a avaliação do atributo como porta de entrada do serviço de saúde encontra-se adequado, mas entende que o acesso às ações e aos serviços efetivados pelas Estratégia Saúde da Família não contemplam a totalidade das necessidades da população assistida, diante da percepção dos usuários oncológicos.

O baixo escore atribuído ao Acesso na perspectiva dos usuários oncológicos pode estar relacionado ao fato de as equipes das Estratégias Saúde da Família trabalharem em dias e horários comerciais e atenderem os usuários somente com agendamento prévio de consulta, com exceção de pequenas urgências. ${ }^{(20)}$ A realização do horário estendido poderia diminuir dificuldades de Acesso e ampliar a utilização dos serviços.

As socializações e as reflexôes no grupo possibilitaram identificar situaçôes vivenciadas e também divergências entre as equipes.

"Eu sou mais do turno único." (TEC. ENF. ESF 1)

"Não é realizado horário estendido, porém sua realizaçáo seria interessante, pois daria para atender a população que não tem acesso ao horário habitual de funcionamento da unidade." (ENF. ESF 2) 
"Nós realizamos alguns turnos estendidos, mas eles são especificos, não é uma rotina." (NUT. ESF 3)

Os profissionais relataram que, entre as lacunas existentes para que se possa efetivar, de fato, o horário estendido, está a não autorização do gestor municipal.

"A gestão municipal nos proibiu de fazer horário estendido, porque os funcionários começaram a ter um banco de horas muito grande." (ENF. ESF 3)

"Nós fizemos um pedido para administração municipal, a unidade mandou um ofício solicitando a possibilidade de realizarmos turno estendido, mas foi negado [...] agora eles nos disseram que estão pensando sobre a possibilidade de efetivarmos." (ENF. ESF 5)

Garantir acesso universal aos usuários nos serviços pode favorecer a resolutividade da atenção à saúde, o que contribui para o atendimento a problemas de saúde crônicos, articulando promoção da saúde e prevenção de doenças. ${ }^{(21)}$ Uma importante ferramenta para fomentar o acesso do usuário oncológico aos serviços na Atenção Primária à Saúde é dispor de horários de atendimento estendidos, pois considera-se este um facilitador do processo de cuidado.

A Longitudinalidade, é definida como a dispensação regular de atenção e seu uso ao longo do tempo, sem que haja, necessariamente, a presença de problemas relacionados à saúde. ${ }^{(8)}$ Ao refletirem sobre o resultado insatisfatório, participantes em convergência entendem que este pode estar relacionado à rotatividade dos profissionais, o que fragiliza a formação do vínculo, no entanto, trata-se de um pressuposto da Estratégia Saúde da Família, fazendo-se necessária sua presença.

"Entendo que esse baixo escore está relacionado à rotatividade dos profissionais, principalmente médicos e enfermeiros [...]." (NUT. ESF 3)

"Além da troca frequente de profissionais, o que também acaba afetando o atributo é a falta de qualidade dos registros em prontuário." (NUT. ENF 4)

Nessa perspectiva, observa-se a importância da fixação de profissionais na unidade de saúde, para estreitar o vínculo entre profissionais de saúde e usuários, pressuposto fundamental da ESF. Ainda, quando se privilegiam registros minuciosos e completos, obtém-se qualidade nas informaçóes registradas no prontuário, o que possibilita tratamentos mais eficazes e contribui para a resolutividade dos problemas de saúde. ${ }^{(22)}$

Trabalhadores em interação perceberam que podem e devem qualificar os registros. Sintetizando reflexôes do grupo, é possível inferir, ainda, que a percepção insatisfatória dos profissionais de saúde acerca da Longitudinalidade pode estar relacionada à ineficiência da contrarreferência do serviço especializado, o que implica na falta de empoderamento da equipe das Estratégias Saúde da Família para auxiliar os usuários nas suas demandas de cuidado.

"Não temos contrarreferência nem do serviço especializado, nem do hospitalar, de lugar nenhum, então não sabemos o que acontece, e os pacientes também não sabem nos dizer, é difícil [...]." (MED. ESF 3)

A aplicabilidade do atributo Longitudinalidade na APS proporciona impactos positivos, tanto para o cuidado do indivíduo, da família e da comunidade, quanto para a efetividade da Rede de Atenção à Saúde. Ainda, é importante salientar que, quando o cuidado é dispensado a usuários com câncer, é essencial que o mesmo seja acompanhado longitudinalmente, bem como sua família, tendo em vista o complexo cenário caracterizado pelo processo de saúde-doença.

O atributo Integralidade é definido como o leque de serviços disponíveis e prestados pelo serviço de Atenção Primária à Saúde, que abarca atividades de caráter biopsicossocial do processo saúde-doença, além de ações de promoção, prevenção, cura e reabilitação. ${ }^{(23)}$ Diante dessa realidade, apesar da importância da Integralidade para garantir a efetividade da Atenção Primária à Saúde, os componentes desse 
atributo alcançaram resultado aquém do esperado. O grupo em interação, por exemplo, expressou, após momentos de reflexáo e socialização, que, dentre seus usuários, o paciente oncológico necessita de uma gama maior de cuidados que, por vezes, a Atenção Primária à Saúde não contempla em sua totalidade.

"Quando a pessoa passa por algum tipo de sofrimento, no caso, o câncer, mesmo ela tendo um bom entendimento sobre as demandas do serviço, ainda, para ela isso não é o suficiente [...]." (ACS. ESF 5)

"Às vezes as necessidades de integralidade do cuidado na percepção do paciente oncológico podem necessitar de maior agilidade e, se fossem de repente entrevistados em uma outra condiçâo de saúde, quem sabe, não avaliariam de forma insatisfatória." (MED. ESF 4)

Ainda, no entendimento compartilhado do grupo, os profissionais ponderaram que, nos diferentes níveis da Rede de Atenção à Saúde, quando é descoberto um novo problema de saúde, como o câncer, para o indivíduo acometido, o acesso aos serviços passa a ser emergencial e a demora nos encaminhamentos relacionada a aspectos burocráticos do sistema, sendo, por vezes, fator desencadeante de anseios, angústia e temores, o que pode, ainda, refletir na insatisfação dos pacientes com o serviço e os profissionais.

"Pacientes com câncer encontram-se em um momento frágil de suas vidas, assim, desde a demora no atendimento, a maneira com que os profissionais atendem essa pessoa, até a forma com que o serviço é ofertado, pode não ser encarado de forma positiva por esses usuários." (MED. ESF 2).

“[...] Eu precisei do serviço especializado uma vez e o que eu pude perceber é que existe falta de organização da gestão. São muitos aspectos burocráticos, alguns até mesmo desnecessários [...]." (ACS. ESF4).

Relatos evidenciaram ainda que, por se tratar de uma doença crônica e complexa, por vezes o cuidado dispensado ao paciente oncológico não transcende os aspectos relacionados à saúde-doença, vindo de encontro ao que é preconizado em relaçáo à atenção à saúde, a qual deve estar orientada para a pessoa, e não somente para a doença. Essa realidade demonstra a necessidade de a Atenção Primária à Saúde ampliar a rede de serviços ofertados, atendendo às necessidades básicas de saúde da população e suas famílias, aliada à oferta de cuidados, como orientaçóes e manejo das condiçôes prevalentes. ${ }^{(24)}$

Referente ao atributo Coordenação, ele é entendido como a articulação entre os diversos serviços e açôes de saúde, de forma que estejam sincronizados e voltados ao alcance de um objetivo, com vistas a ofertar ao usuário um conjunto de serviços e informaçóes que respondam a suas necessidades de saúde, de forma integrada, por meio de diferentes pontos da Rede de Atenção à Saúde. ${ }^{(20)}$

A integração entre os serviços de diferentes níveis de complexidade, de modo a possibilitar o acesso tanto à Atenção Primária à Saúde como a atendimentos especializados e de alta complexidade tecnológica, é responsabilidade fundamental do SUS. ${ }^{(21)}$

"A Atenção Primária à Saúde deveria coordenar o cuidado, mas, às vezes, faltam as ferramentas adequadas para que a assistência seja efetiva, porque, quando não temos a contrarreferência do paciente oncológico, o cuidado náo é realizado baseado na real condição do individuo." (ENF. ESF 3)

Profissionais em interação demonstraram-se implicados no processo de qualificação, expressam suas percepçóes e sugerem estratégias de intervençóes para superaçáo dos problemas listados. De acordo com reflexões das equipes das Estratégias Saúde da Família, eles entendem, concordam e acolhem a insatisfação dos usuários oncológicos, no que se refere a esse atributo e, novamente, referenciam a incipiência na contrarreferência do serviço especializado para a APS.

"[...] Se o paciente vê como insatisfatório, acho que ele avaliou bem, porque nós temos que pedir para ele nos trazer informaçóes sobre seu atendimento 
no serviço de alta complexidade, e isso é errado [...]." (ENF. ESF 3)

"Nós pressupomos que a referência funciona, porque, para qualquer especialista que encaminhamos os usuários, temos que enviar junto o histórico, exames e outros itens, mas a contrarreferência não existe." (ACS. ESF 4)

Nesse sentido, a Coordenação do Cuidado com paciente oncológico precisa ir além do encaminhamento, visando promover a comunicaçáo entre os profissionais e os serviços, com garantia de referência e contrarreferência, ou seja, preconiza a integração de cuidados e o compartilhamento das informaçóes geradas pelos profissionais na oferta de serviços, desde a porta de entrada e de volta para ela, de modo que ocorra a continuidade do cuidado e possibilite a resolutividade do cuidado na Atenção Primária à Saúde. ${ }^{(16)}$

O atributo Orientação Familiar está centrado na avaliação das necessidades individuais, que leva em consideraçáo o contexto familiar e seu potencial de cuidado à saúde. ${ }^{(8)}$ As discussōes e reflexóes durante as açôes educativas possibilitaram refletir situaçôes vivenciadas, bem como identificar fragilidades para que seja, de fato, implementado tal atributo na Atenção Primária à Saúde.

"Conseguimos realizar um bom acompanhamento com os pacientes que dão abertura para a equipe de saúde, mas são poucos." (ACS. ESF 4)

"Quando se descobre a doença e os usuários passam a realizar o tratamento, a referência deles passa a ser o serviço especializado nesse momento e não retornam para a Estratégia Saúde da Família." (NUT. ESF 3)

"Temos algumas famílias que banalizam a doença de seu familiar com câncer e acabam náo dando tanta importância e buscam a assistência no serviço especializado." (ENF. ESF 5)

A partir das reflexóes dos trabalhadores em saúde, pode-se ponderar que, apesar da estratégia de
Atenção Primária à Saúde no Brasil ser a Saúde da Família, o que se observa, a partir destes resultados, é que o serviço se encontra centrado na atenção individual do usuário, privilegiando a doença e as demandas dela advindas, sem o enquadrar no contexto familiar que está inserido.

Ainda, a família, como foco das Estratégias Saúde da Família, deve ser compreendida de forma integral em seu espaço social, uma vez que é na família que ocorrem interaçóes e conflitos que interferem diretamente na vida das pessoas. ${ }^{(25)}$

A Orientação Comunitária é definida como o reconhecimento das necessidades por parte do serviço de saúde, por meio de dados epidemiológicos e do contato direto com a comunidade, bem como do planejamento e da avaliaçáo do serviço. ${ }^{(26)}$ Resultados insatisfatórios dos atributos derivados da Atenção Primária à Saúde, na ótica dos usuários oncológicos, preocupam, tendo em vista que representam elementos fundamentais para o fortalecimento do vínculo entre serviço de saúde, famílias e comunidade, considerando a família como o eixo do cuidado e a Estratégia Saúde da Família como modelo de saúde vigente. ${ }^{(25)}$

Nesse sentido, os partícipes manifestaram que a visão insatisfatória dos usuários oncológicos acerca desse atributo pode estar relacionada a aspectos específicos.

"Autoavaliando-nos, os pacientes estão certos e, nós, profissionais, estamos errados, porque antigamente nós não tínhamos o conhecimento das caracteristicas da nossa comunidade, hoje temos." (ENF. ESF 5)

"Acho que o problema é a falta de corresponsabilização dos usuários no que se refere à parte de controle social, eles não procuram saber que é dever deles participar, montar um conselho de saúde local [...]." (ENF. ESF 3)

O grupo em interação aludiu a pouca inserção comunitária aliada à não participação social na Atençáo Primária à Saúde como fator relacionado às fragilidades desse atributo. A incipiente participação em questôes de interesse populacional acarreta no desconheci- 
mento das necessidades dos habitantes do território, o que resulta em um cuidado fragmentado. ${ }^{(27)}$

Dessa forma, um entendimento das características de saúde na comunidade e dos recursos disponíveis fornece uma forma mais extensa de avaliar as necessidades, do que uma abordagem baseada apenas nas interaçóes com os pacientes ou com suas famílias. ${ }^{(26)}$ Tais princípios são condizentes com os propostos da Estratégia Saúde da Família, que tem como objetivo romper com o comportamento passivo das equipes de saúde e estender suas açóes para toda a comunidade.

Nessa perspectiva, a presença e a extensão dos atributos da Atenção Primária à Saúde em sua totalidade, permitem a consolidação dos serviços de saúde efetivados por meio dos trabalhadores atuantes nas Estratégias Saúde da Família. Dessa forma, os serviços de Atenção Primária à Saúde orientados para seus atributos resultam em melhores indicadores de saúde, maior eficiência no fluxo dos usuários dentro do sistema, tratamento mais efetivo das condiçôes crônicas, cuidado resolutivo, maior satisfação dos usuários e diminuição das desigualdades de acesso aos serviços de saúde. ${ }^{(25)}$

A fim de garantir a efetivação das ações desempenhadas pelos trabalhadores da Atenção Primária à Saúde, faz-se necessária a atualização contínua e permanente, por meio da construção do conhecimento. Assim, entende-se que, na execução de estratégias educativas, é possível recolocar saberes e repensar práticas consolidadas. ${ }^{(28)}$

Em síntese, nas pactuaçóes realizadas com vistas à efetivação de um cuidado qualificado ao paciente oncológico, profissionais em interação entenderam que faz-se necessário que a assistência a esse usuário seja realizada por meio da Rede de Atenção à Saúde, pautada nas atribuiçôes específicas da Atenção Primária à Saúde, com vistas a garantir a resolutividade da atenção e a efetividade do atendimento dispensado. Ainda, inferiram ser imprescindível re(conhecer) o itinerário terapêutico do usuário, percorrido nos diversos níveis assistenciais durante o processo de diagnóstico, tratamento, cura e reabilitação do câncer. Somente assim deve ser possível assistir as demandas de saúde-doença do indivíduo em sua totalidade.

\section{Conclusão}

A análise dos resultados permite inferir que implementar uma prática educativa possibilita trazer em pauta aspectos importantes, que integram a assistência e que, por vezes, constituem-se em rotina e explicitam fragilidades, as quais necessitam ser superadas na perspectiva de dispensar assistência integral e de qualidade aos usuários, em especial aos pacientes oncológicos. Desse modo, a utilização da metodologia pautada na pesquisa-ação teve potencial de produzir nos trabalhadores das Estratégias Saúde da Família reflexões sobre o fazer assistencial e, na mesma medida, identificar fragilidades, o que implicou em analisá-las e em buscar superá-las. $\mathrm{O}$ desenvolvimento da prática educativa estimulou o diálogo da equipe, no intuito de abstrair saberes e compreensóes da assistência efetivada ao paciente oncológico e, diante da fragilidade identificada, entendeu-se necessário compreendê-la e, na mesma medida, propor meios para o fortalecimento da atenção em saúde ao paciente oncológico. Ainda, a socialização possibilitou fortalecer a corresponsabilização da equipe com o cuidado aos indivíduos de sua área de adscrição, independente da formação. Com isso, torna-se indispensável que equipes de saúde desenvolvam ações de cuidado a esses pacientes, bem como identifiquem a incidência desses indivíduos em seu território de abrangência. Pontuase, ainda, a necessidade da implementação de ferramentas que evidenciem a presença dos princípios que norteiam o serviço de saúde, com vistas a contemplar os atributos da Atenção Primária à Saúde. Logo, é importante e necessário avaliar os serviços oferecidos aos indivíduos, de forma a manter sua qualificação contínua. Destacam-se, ainda, que, as oficinas fomentaram um olhar ampliado ao cuidado efetivado na Atenção Primária à Saúde, bem como a percepção das diferentes equipes de Estratégia Saúde da Família em relação ao cuidado ao usuário oncológico dispensado em todos os pontos da Rede de Atenção à Saúde, para propiciar o fortalecimento e a corresponsabilização das equipes com o cuidado com os indivíduos de sua área de adscrição. 


\section{Agradecimentos}

Ao Conselho Nacional de Desenvolvimento Científico e Tecnológico que pelo Programa Institucional de Bolsas de Iniciação Científica (PIBIC) concedeu bolsas de iniciação científica, que permitiu a coleta de dados, por meio dos bolsistas.

\section{Colaborações}

Trindade LF, Kolankiewicz ACB, Bandeira LR, Rodrigues CD, Zeitoune RCG e Loro MMM contribuíram com a concepção do estudo, análise e interpretação dos dados, redação do artigo, revisão crítica relevante do conteúdo intelectual e aprovação da versão final a ser publicada.

\section{Referências}

1. Facchini LA, Tomasi E, Dilélio AS. Quality of Primary Health Care in Brazil: advances, challenges and perspectives. Saúde Debate. 2018;42(Spe 1):208-23.

2. Ribeiro M, Napoleão Albuquerque I, Cunha I, Mayorga F, Ximenes Neto F, Silveira N. Organização do cuidado às condições crônicas na atenção primária à saúde de Sobral-CE: avaliação de processo na perspectiva de gestores. APS. 2019;1(1):29-38

3. Rosa LM, Souza Al, Anders JC, Silva RN, Silva GS, Fontão MC. Oncology nursing care and qualification demands in primary health care. Cogitare Enferm. 2017;4(22):e51607.

4. Panis C, Kawasaki ACB, Pascotto CR, Justina EY, Vicentini GE, Lucio LC, et al. Critical review of cancer mortality using hospital records and potential years of life lost. einstein (São Paulo). 2018;16(1):eA04018.

5. Lopes AB, Guimarães IV, Melo IM, Teixeira LS, Silva SV, Silva MH, et al. Fatores modificadores da qualidade de vida em pacientes oncológicos em tratamento quimioterápico. Rev Med Minas Gerais. 2016;26(Supl 3):41-6.

6. Visentin A, Mantovani MF, Kalinke LP, Boller S, Sarquis LM. Palliative therapy in adults with cancer: a cross-sectional study. Rev Bras Enferm. 2018;71(2):252-8.

7. Oliveira Friestino JK, Silveira Corrêa CR, de Carvalho Moreira Filho D. Percepções dos profissionais sobre o diagnóstico precoce do câncer infantojuvenil na Atenção Primária à Saúde. Rev Bras Cancerol. 2017;63(4):265-72.

8. Starfield B. Atenção Primária: equilíbrio entre necessidades de saúde, serviços e tecnologias [Internet]. Brasília: UNESCO; Ministério da Saúde; 2002 [citado 2019 Jul 21]. Disponível em: http://bvsms.saude. gov.br/bvs/publicacoes/atencao_primaria_p1.pdf

9. Brasil. Ministério da Saúde. Secretaria de Atenção em Saúde. Departamento de Atenção Básica. Manual do Instrumento de Avaliação da Atenção Primária à Saúde [Internet]. Brasília (DF): Ministério da
Saúde; 2010 [citado 2019 Jun 20]. Disponível em: http://bvsms.saude. gov.br/bvs/publicacoes/manual_avaliacao_pcatool_brasil.pdf

10. Kolankiewicz AC, Loro MM, Magnago TS, Rosanelli CL, Domenico EB. Demands of cancer patients care: proposal of intervention for convergence and educational practice. Rev Enferm UFPE. 2015;9(12):1370-5.

11. Loro MM, Bittencourt VL, Zeitoune RC. Assistance convergent research: nursing team sharing occupational risk Knowledge and proposing interventions. Rev Min Enferm. 2017;21:e1044.

12. Leiyushi L, Starfield B, Xu J. Validating the adult primary care assessment tool. J Fam Pract. 2001;50(2):161-75.

13. Harzheim E, Oliveira MM, Agostinho MR, Hauser L, Stein AT, Gonçalves MR, et al. Validação do instrumento de avaliação da atenção primária à saúde: PCATool-Brasil adultos. Rev Bras Med Fam Comunidade. 2013;8(29):274-8.

14. Hauser L, Castro RC, Vigo A, Trindade TG, Gonçalves MR, Stein AT, et al. Tradução, adaptação, validade e medidas de fidedignidade do Instrumento de Avaliação da Atenção Primária à Saúde (PCATool) no Brasil: versão profissionais de saúde. Rev Bras Med Fam Comunidade. 2013;8(29):244-55.

15. Bardin L. Análise de conteúdo. Lisboa: Edições 70; 2011.

16. Santos FP, Acioli S, Rodrigues VP, Machado JC, Souza MS, Couto TA. Nurse care practices in the Family Health Strategy. Rev Enferm UFPE. 2016;69(6):1124-31.

17. Késia A, Pereira IF, Oliveira RM, Silva RI. A pesquisa-ação nas publicações da Revista Brasileira de Educação (2016-2018). Res Soc Dev. 2019;8(10):1-17.

18. Cordeiro L, Soares CB. Work Process in Primary Health Care: action research with Community Health Workers. Ciênc Saúde Coletiva. 2015;20(11):3581-8.

19. Bispo GM, Rodrigues EM, Carvalho AC, Lisboa KW, Freitas RW, Damasceno MM. Assessment of access to first contact in the perspective of professionals. Rev Bras Enferm. 2020;73(3):1-7.

20. Rabelo AL, Lacerda RA, Rocha ES, Gagno J, Fausto MC, Gonçalves MJ. Care coordination and longitudinality in primary health care in the Brazilian Amazon. Rev Bras Enferm. 2020;73(3):e20180841.

21. Silva AN, Silva SA, Silva AR, Araújo TM, Rebouças CB, Nogueira LT. Primary care assessment from a male population perspective. Rev Bras Enferm. 2018;71(2):236-43.

22. Araujo JP, Viera CS, Oliveira BR, Gaiva MA, Rodrigues RM. Assessment of the essential attributes of Primary Health Care for children. Rev Bras Enferm. 2018;71(Suppl 3):1366-72.

23. Gontijo TL, Duarte AG, Guimarães EA, Silva J. Avaliação da atenção primária: o ponto de vista de usuários. Saúde Debate. 2017;41(114):741-52.

24. Gasparini MF, Furtado JP. Longitudinality and comprehensiveness on the More Doctors Program: an evaluative study. Saúde Debate. 2019;43(120):30-42.

25. Batista VC, Ribeiro LC, Ribeiro CD, Paula FA, Araújo A. Avaliação dos atributos da atenção Primária à saúde segundo os profissionais de saúde da família. SANARE. 2016;15(2):87-93.

26. Reichert AP, Leônico AB, Toso BR, Santos NC, Vaz EM, Collet N. Family and community orientation in children's primary healthcare. Ciênc Saúde Coletiva. 2016;21(1):119-27.

27. Bandeira LR, Kolankiewicz AC, Alievi MF, Trindade LF, Loro MM. Fragmented comprehensive health care for ostomized person in the health care network. Esc Anna Nery. 2020;24(3):e20190297.

28. Loro MM, Zeitoune RC. Collective strategy for facing occupational risks of a nursing team. Rev Esc Enferm USP. 2017;51:e03205. 